\title{
Application of a Genetic Algorithm for Minimum Zone Method of Flatness
}

\author{
Balázs Mikó, Soma Manó Szabó, Ágota Drégelyi-Kiss \\ Institute of Material and Manufacturing Science, Óbuda University \\ Népszínház u. 8, H-1081 Budapest, Hungary \\ e-mail: miko.balazs@bgk.uni-obuda.hu,dregelyi.agota@bgk.uni-obuda.hu
}

\begin{abstract}
The geometric product specification (GPS), has an increasing importance in machine design, manufacturing and measuring. However, standards describe the interpretation of the different kinds of form and position tolerances; there are several methods in measuring, how these deviations can be evaluated. In this article the minimum zone method $(M Z)$ is presented in case of flatness error by coordinate measuring device. Different search algorithms can be applied during the implementation, in order to solve the geometric problem of flatness evaluation. In this article, the genetic algorithm is investigated and compared with hill climbing algorithms. The optimization of the parameters of the genetic algorithm is also presented.
\end{abstract}

Keywords: geometric tolerances; coordinate measuring method; flatness; minimum zone method; genetic algorithm

\section{Introduction}

A plane surface is an often used geometric feature, in the machine design. The accuracy of a plane can be defined by many aspects, but the set of geometric deviations can ensure the most sophisticated description. The flatness error describes the deviation of a flat surface from the theoretical plane. The flatness is a type of geometric error and the flatness tolerance defines the permissible level of this error. The flatness tolerance is defined by standards [1] [2]. The application of geometric tolerances has several aspects. The first is the notation in the shop drawing, the second is the functional justification, the third is the manufacturing aspect and the fourth is the measuring aspect. The standards describe the first level only.

The flatness error is the distance of two parallel planes, which limit the produced flat surface. The two parallel planes have 3 degree of freedom (DOF), one linear, in perpendicular direction and two angular DOFs, in the horizontal plane. During the calculation of flatness error, the position of the parallel planes should be defined. 
The geometric error of the different surfaces can be measured by coordinate measuring machines. There are several parameters, which defined the uncertainty of the measuring process [3]. The calculation method of the geometric errors is one of the key parameter.
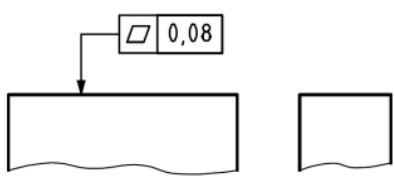

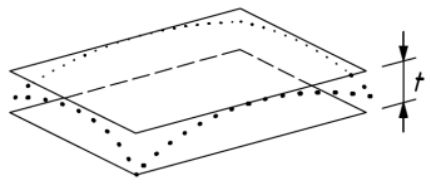

Figure 1

Indication and implementation of flatness based on ISO 1101

In current literature, there are several methods for solving the problem of evaluate flatness error, like regression method, envelope method, minimum zone method, but in the current article the minimum zone method (MZM) is investigated (applied). In case of MZM method, the position and orientation of the control planes are defined considering the minimal distance between the planes. This requires a recursive method, where the best position is reached by step by step.

Abdulshahed et al. [4] and Yang et al. [5] classified the optimization algorithms as deterministic (simplex method, downhill method, Newton-Raphson method, least square method (LSM), convex hull algorithm) and stochastic methods (Figure 2). Inside meta-heuristic stochastic method, the genetic algorithms (GA) and the different kind of particle swarm algorithms (PSO) are the most popular methods.

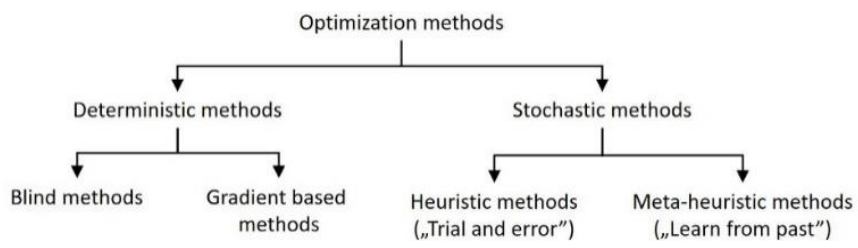

Figure 2

Classification of optimization methods

In case of evaluation of flatness, a wide range of optimization method can be used for determine the minimum zone of flatness. Kanada and Suzuki [6] compared downhill simplex method, with repetitive bracketing method. 8400 points were measured at test parts, which were machined by shaping, milling and hand finishing. The downhill method was found to be faster. Samuel and Shunmugam [7] and Hermann [8] investigated convex hull method. Abdulshaded et al. [4] a kind of swarm algorithm applied: the Cuckoo search optimization. Yang et al. [5] compared five types of algorithms: LSM, GA, PSO, teaching-learning based algorithm (TLBO) and adaptive hybrid TLBO. Based on different point clouds (number of points 25 and 32), the AH-TLBO algorithm showed faster regression and smaller flatness values. 
Tseng [9] applied genetic algorithm (GA) for minimum zone flatness calculation. Arithmetic crossover and non-uniform mutation were applied. The size of the population was doubled, but other parameters are not published. The individuals were selected based on the roulette wheel method, when the selection is random, but the probability is proportion with the fitness. Based on literature based point clouds, the proposed GA method ensured better results than least square method or convex hull method. Wang et al. [10] presents a differential evolutionary algorithm (DE) for flatness calculation by minimum zone method. The population consists of 20 individuals in the implementation, the best individuals were selected for crossover and after 100 iterations the process was stopped. The authors found, that the proposed algorithm has the advantages of simplicity and flexibility. Wen et al. [11] applied an improved genetic algorithm (IGA) for minimum zone flatness evaluation, which ensures shorter processing time by realcode implementation. The blend crossover function was applied, when parents of a new individual was selected randomly. The investigated point cloud contained 20 points. The size of the population was 20 , and during one iteration 20 new individuals were generated. The iteration was stopped at 200 cycles. Comparing with the least square method, the results were better. Khan and Ma [12] presents a real coded efficient genetic algorithm (EGA) in case of flatness assessment based on minimum zone method. The proposed algorithm converged in less than twenty generations. The implementation was performed in Matlab, the size of the population was 60 , and the $50 \%$ of the population was regenerated by crossover in every generation. $10 \%$ of the generation was modified by a decreasing random mutation function.

During the previous research [13] four hill-climbing algorithms were compared, but they were single point methods, one solution was modified in several steps.

In this research, a genetic algorithm solution was implemented and investigated. The genetic algorithm is a stochastic optimization method based on the principle of population evolution [14]. An individual - a member of the population - is a potential solution of the problem, and through the evolution process the best individual will be the final result. The representation of the individuals can be binary or real-code, depending on the investigated problem. During the evolution, three operators are applied: selection, crossover and mutation. The selection for crossover of mutation can be random, statistical (like roulette wheel selection) or elitist (select the best). The crossover function creates new individuals based on selected individuals (parents), and the mutation function modifies the selected individual. The implementation of the genetic algorithm can be very different [14] [15], but based on the literature during the implementation the main questions are the following:

- How can the individual be described?

- How large population is required?

- What is the fitness function? 
- How can the crossover and mutation be interpreted?

- How the individuals can be selected?

- How many individuals are made by crossover?

- How many individuals are modified by mutation?

The aim of the article is to present an implementation of the genetic algorithm in case of the evaluation of the flatness error and to investigate the effect of the different parameters of the algorithm on the performance of the algorithm. The optimization of the parameters is presented also.

\section{GA Implementation}

The flatness error of a measured point cloud can be defined as the difference of the closest and the farthest points from a reference plane. This definition harmonizes with the definition of the standard and realizes the minimum zone method. From the viewpoint of the genetic algorithm, the position of the reference plane means one solution of the problem, so the population consists of a set of reference planes. An individual means the $\mathrm{N}_{X}, \mathrm{~N}_{Y}$ and $\mathrm{N}_{Z}$ coordinates of the normal vector of a reference plane.

The fitness function of the genetic algorithm is the flatness error, which can be calculated by the normal vector and the set of points of plane surface. A plane can be defined by a point $\left(\underline{P}_{o}\right)$ and the normal vector $(\underline{N})$. The $P_{o}$ point hasn't got an importance in this application, so it can be $(0 ; 0 ; 0)$. The distance of a point $\left(\underline{P}_{i}\right)$ is calculated by equation (1):

$$
D_{i}=\frac{N_{x} \cdot\left(P_{0 x}-P_{i x}\right)+N_{z} \cdot\left(P_{0 y}-P_{i y}\right)+N_{z} \cdot\left(P_{0 z}-P_{i z}\right)}{\sqrt{N_{x}^{2}+N_{y}^{2}+N_{z}^{2}}}
$$

Based on the minimum zone method, the flatness error is the difference between the farthest and the closest point (2) on the surface:

$$
F L=\operatorname{Max}\left(D_{i}\right)-\operatorname{Min}\left(D_{i}\right)
$$

Individuals, which have the best fitness values, can generate new individuals by crossover function. In this case, the crossover function is implemented as a linear combination of selected normal vectors. The new normal vector is on the connection line of parent vectors. The position is selected randomly and the $25 \%$ external section is permitted too (Figure 3a). In case of mutation function, the normal vector is modified by $\pm 10 \%$ randomly (Figure $3 a$ ). 


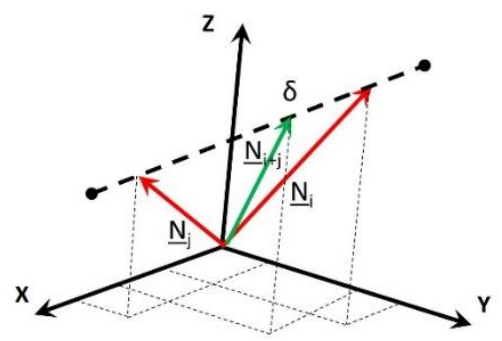

a)

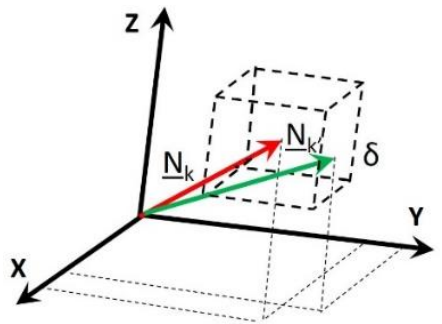

b)

Figure 3

Implementation of crossover (a) and mutation (b) function

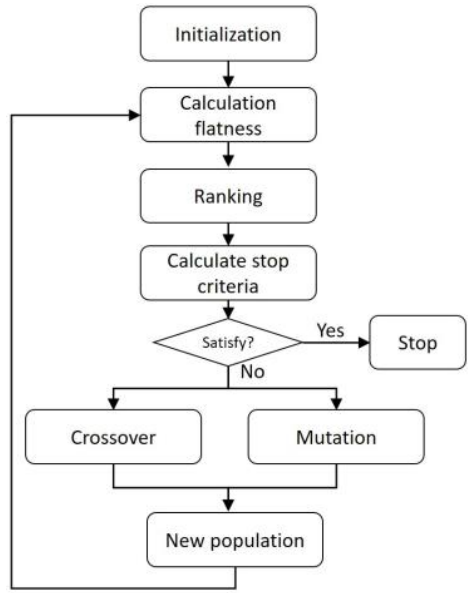

Figure 4

The GA process

During the preliminary investigation, the main questions were the size of the population, the number of new individuals, which can be generated by crossover and the number of individuals, which can be modified by mutation. Figure 4 shows the investigated algorithm. The initial population (a set of normal vector of the reference planes) is generated randomly during the initialization, the $N_{x}$ and $N_{y}$ coordinates were changed, the $N_{z}$ was 1 in the current implementation. Then the flatness values are calculated for every individual (reference plane). Based on it, the ranking can be performed. Then the stop criterion is investigated: if the difference between the best and the worst flatness values is smaller than 0.00001 $\mathrm{mm}$, or the number of iteration is 1000 , the process stops. Otherwise, the crossover and the mutation functions are executed, and the process starts again with the new (modified) population. The algorithm was implemented in Free Pascal 1.0.12. 


\section{Test Environment}

The performance of the genetic algorithm is defined by the main parameters, as the size of the population, the number of new individuals and the number of modified individuals. The optimization of these parameters improve the efficiency of the algorithm. In order to optimization several sets of parameters were defined and the results and performance were analysed. A full factorial plan was used for generate parameter sets, because of the fast calculation process. The number of variations was 4 × 8 × $6=192$, and every set was repeated 25 times.

Three factors were varied:

- The size of the population. Pop $=15-20-25-30$

- The number of new individuals: Cro $=1-2-3-4-5-6-7-8$

- The number of modified individuals: Mut $=1-2-3-4-5-6$

The algorithm was tested on six machined flat surfaces of the same dimensions $175 \mathrm{~mm} \times 155 \mathrm{~mm}$. The test surfaces were machined using different technologies, methods and machine tools (Table 1). Surfaces \#1 and \#2 were machined in a conventional and $\mathrm{CNC}$ milling machine by zig-zag strategy, with the same cutting speed and feed per tooth. Surfaces \#3 and \#4 were machined by face turning with the same parameters, except the feed. As for \#5 and \#6 surfaces, face-milling technology was applied with the same tool and parameters, and with different tool path strategies.

Table 1

Machining conditions of test samples

\begin{tabular}{|l|c|c|c|c|c|c|}
\hline Surface ID & Sf\#1 & Sf\#2 & Sf\#3 & Sf\#4 & Sf\#5 & Sf\#6 \\
\hline Method & \multicolumn{2}{|c|}{ Face milling } & \multicolumn{2}{|c|}{ Face turning } & \multicolumn{2}{|c|}{ Face milling } \\
\hline Strategy & \multicolumn{2}{|c|}{ Zig-Zag } & - & Zig-Zag & Spiral \\
\hline Machine tool & \multicolumn{2}{|c|}{ UF-231 } & $\begin{array}{c}\text { MAZAK } \\
\text { A410-II }\end{array}$ & E400-1000 & MAZAK A410-II \\
\hline Type & Manual & CNC & Manual & CNC \\
\hline $\mathrm{D}_{\mathrm{c}}[\mathrm{mm}]$ & 80 & 50 & - & 63 \\
\hline $\mathrm{z}[-]$ & 7 & 4 & \multicolumn{2}{|c|}{1} & 6 \\
\hline $\mathrm{v}_{\mathrm{c}}[\mathrm{m} / \mathrm{min}]$ & \multicolumn{2}{|c|}{60} & \multicolumn{2}{|c|}{$(100)$} & 180 \\
\hline $\mathrm{n}[1 / \mathrm{min}]$ & 240 & 382 & \multicolumn{2}{|c|}{190} & 910 \\
\hline $\mathrm{f} ; \mathrm{f}_{\mathrm{z}}[\mathrm{mm}]$ & \multicolumn{2}{|c|}{0.046} & 0.6 & 0.2 & 0.09 \\
\hline $\mathrm{v}_{\mathrm{f}}[\mathrm{mm} / \mathrm{min}]$ & \multicolumn{2}{|c|}{78} & 70 & 115 & 40 & 490 \\
\hline $\mathrm{a}_{\mathrm{p}}[\mathrm{mm}]$ & \multicolumn{2}{|c|}{1} & \multicolumn{2}{|c|}{0.5} & 1 \\
\hline $\mathrm{a}_{\mathrm{e}}[\mathrm{mm}]$ & 40 & 25 & - & 31.5 \\
\hline
\end{tabular}

$D_{c}-$ Cutting tool diameter; $z-$ Number of teeth; $v_{c}-$ Cutting speed;

$n$-Spindle speed; $f, f_{z}$-feed, feed per tooth; $v_{f}$-feed speed;

$a_{p}$-depth of cut; $a_{e}-$ width of cut 


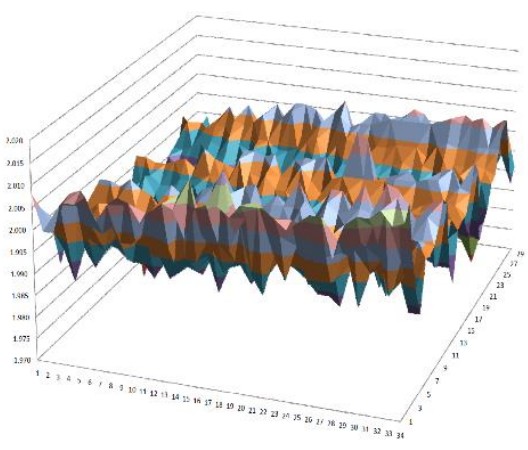

Sf\#1

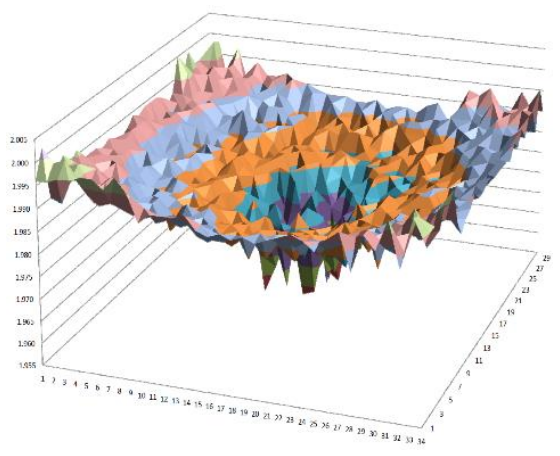

Sf\#3

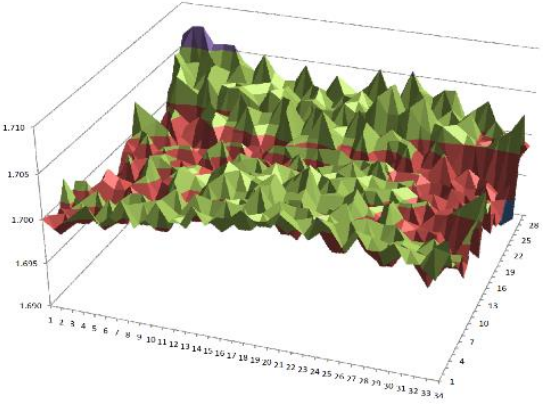

Sf\#5

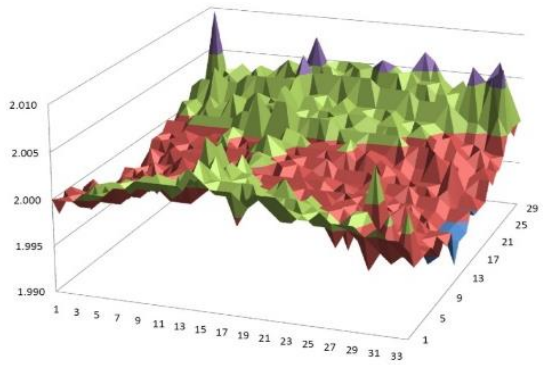

Sf\#2

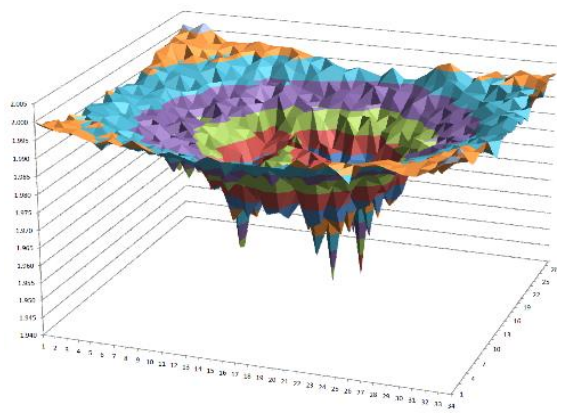

Sf\#4

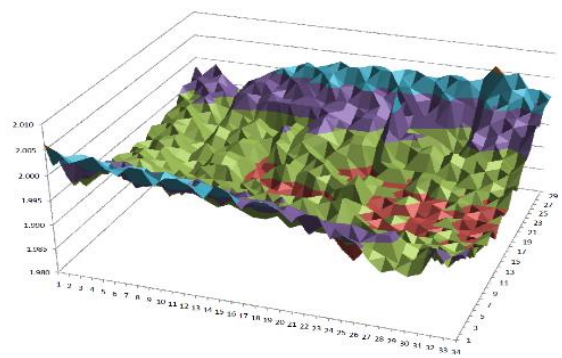

Sf\#6

Figure 5

Maps of measured points in case of 6 test surfaces

The coordinate values of the investigated flat surfaces were measured with Mitutoyo Crysta-Plus 544 coordinate measuring machine. The measurement was performed in a discrete sampling mode with a contact probe (tip diameter is 3 $\mathrm{mm})$. Sampling was carried out in 1020 uniformly distributed points on the 
examined surface. The reference values of the flatness error were calculated by Kotem SurfaceProfile v5. The reference values can be seen in Table 2.

Table 2

Reference values of flatness in $\mathrm{mm}$

\begin{tabular}{|l|c|c|c|c|c|c|}
\hline & Sf\#1 & Sf\#2 & Sf\#3 & Sf\#4 & Sf\#5 & Sf\#6 \\
\hline$F L \_R e f[m m]$ & 0.0343 & 0.0127 & 0.0427 & 0.0572 & 0.0124 & 0.0204 \\
\hline
\end{tabular}

Figure 5 shows the map of coordinate values of six specimens. The different nature of the surfaces, related to the machining technology, can be observed.

The total number of data for optimization was $192 \times 25 \times 6=28.800$. The marking of the different cases is the next: Sf\#4-20-4-6 means, the test was performed in for the data of Sf\#4 test surface, the population consists of 20 individuals, 4 individuals were generated by crossover, and 6 individuals were modified by mutation.

The following parameters were calculated during the test:

- The value of flatness

- The average, the minimum and the maximum values, and the standard deviation of the flatness value of 25 repetitions

- The number of iteration till the stop condition

- The average, the minimum and the maximum values, and the standard deviation

- The ratio of the minimum and the average flatness to the reference values

\section{Iteration Process and Results}

As mentioned before in the current implementation the value of the $Z$ coordinate of the normal vector of a reference plane is constant $\left(N_{z}=1\right)$. During the preliminary phase, the results of test runs were analysed in case of Sf\#6. 15 individuals were generated, the number of crossover is changed from 1 to 8 and the number of mutation is changed from 1 to 6 . The results were calculated based on 25 repeated runs. The results were analysed by MiniTab v14 software (Figure $6)$.

The $\mathrm{Z}$ parameter shows the differences. When all coordinates were changed, the $Z$ $=O$ (3D), and only if the $N_{x}$ and $N_{y}$ were changed (2D), then $Z=1$. As main effects plots show, in case of $Z=1$ the average value of the 25 repeated runs is closer to the reference value $(A v-\operatorname{Ref} \rightarrow 0)$, and the standard deviation of the flatness data is smaller. The standard deviation shows, then the repetition causes smaller difference in the flatness values, the algorithm has better repeatability. 
Nevertheless, more iterations are required, until the stop condition, so the calculation need more time.

Investigating the changing of the flatness values during the iteration in case of $3 \mathrm{D}$ solution the value of the best individual changed just a little and the worst individual evolves close to the first. In case of 2D solution, the first and the worst individuals can evolve too, but it takes more generation (iteration).

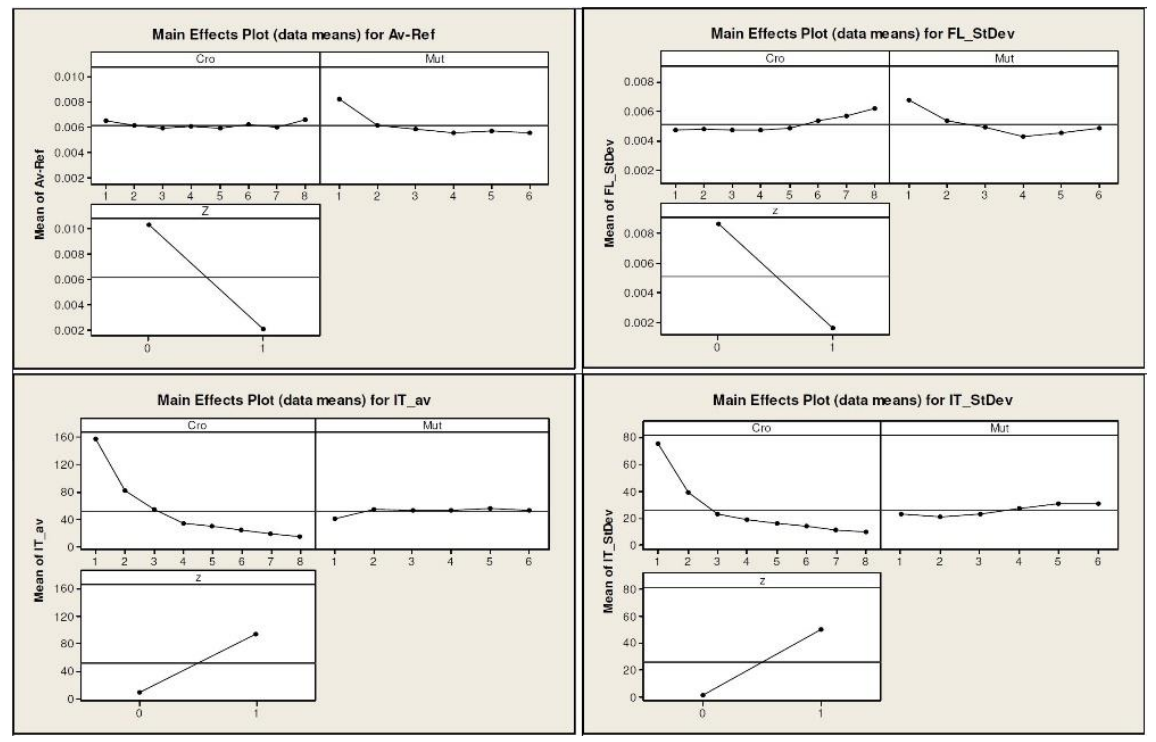

Figure 6

Main effects plot for 2D/3D comparison (Sf\#6; Pop=15)

The Figure 7 shows the distribution of the individuals at the initial and the final step. As it seems, the initial population cover an area, but at final step, the individuals there are in same point. The final position is different in these two cases, because of the randomly generated initial positions. In case of 30 points, 15 points are same, as in the first example (Figure 7a) and one crossover and one mutation were applied. The flatness value in case of 15 populations is $0.0212 \mathrm{~mm}$ and it was reached at 294 iteration steps. In case of 30 individuals, the flatness is $0.0209 \mathrm{~mm}$ and 122 steps were performed.

During the iteration process, the flatness value of the best and the worst individuals is changed gradually. The process stops when the difference between the two values is less than $10 \mathrm{E}-5 \mathrm{~mm}$ (stop condition). 


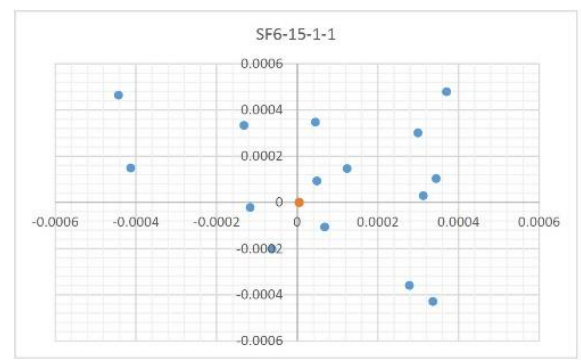

a)

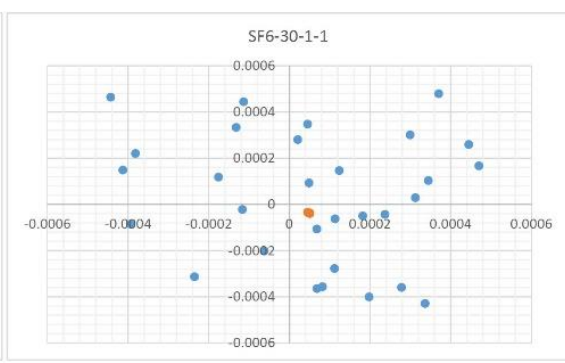

b)

Figure 7

Initial and final population in case of Sf\#6-15-1-1 and Sf\#6-30-1-1

As the Figure 8 shows, the changing of the values has different speed in case of two examples. Of course, the other individuals change too. However, the initial populations cover a very small area $(0.001 \times 0.001 \mathrm{~mm})$, the difference in initial flatness values larger than $0.1 \mathrm{~mm}$.

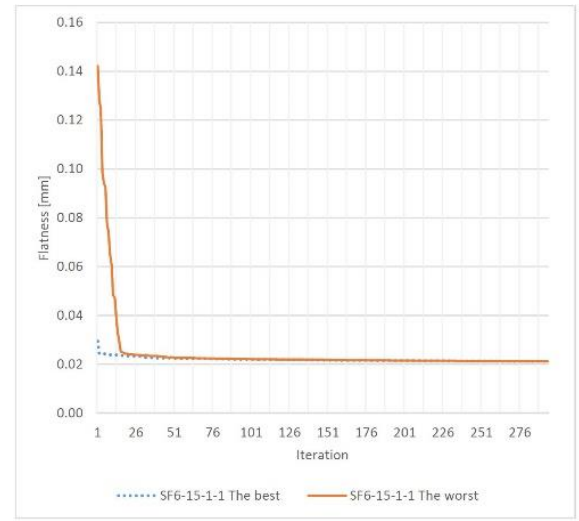

a)

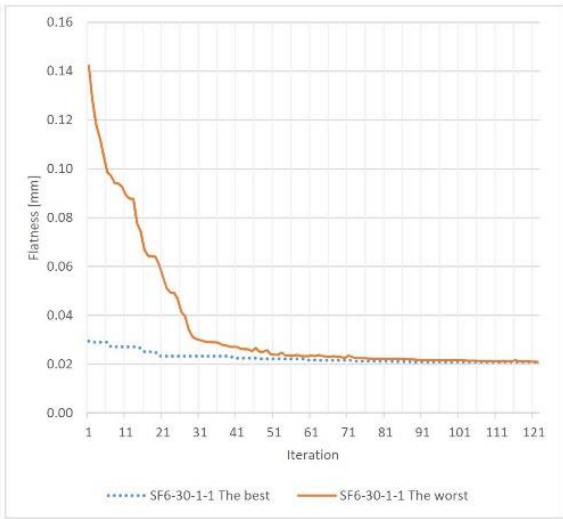

b)

Figure 8

The best and the worst flatness values in case of Sf\#6-15-1-1 and Sf\#6-30-1-1

In the previous research [13] three hill-climbing algorithms were compared. The hill climbing (HC) algorithm is an iterative search algorithm. The HC find the best set of parameters step by step, and in every iteration choose the best neighbour. The difference between the three compared algorithms is the definition of the distance of neighbours. In case of $\mathrm{HC} 1$ random distance was used, $\mathrm{HC} 2$ applied a decreasing random distance and HC3 used fix distance. The results can be seen on the Figure 9. The "GA" means the minimum flatness value from the test runs, so in case of different test surfaces different GA parameters were used. Based on the diagrams the GA solution gives the best results comparing with the reference value, but the aim of this research is to find the GA parameters, which can work properly in every test surfaces. 


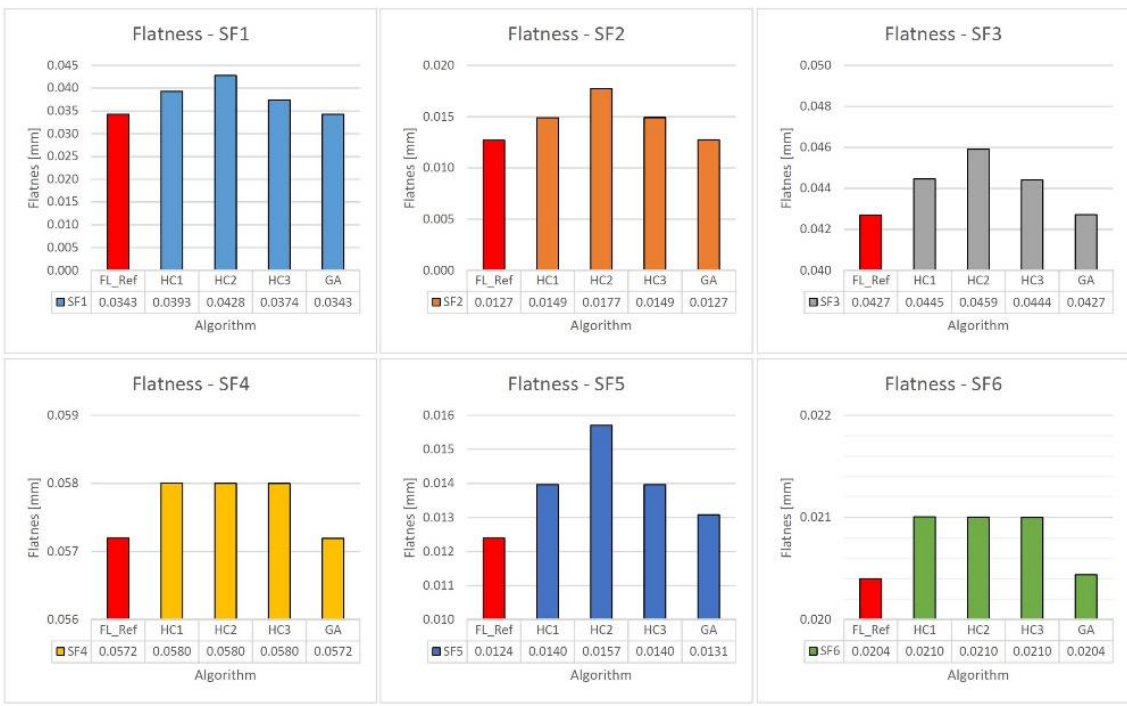

Figure 9

Comparison of the results of different algorithms

\section{Investigation and Optimization of GA Parameters}

The effect of the size of the population (Pop), the number of new individuals (Cro) and the number of modified individuals (Mut) was investigated by main effects plot (Figure 10). The Minitab v14 was used for the analyses.

In case of difference of minimum and reference value (Min-Ref) and the difference of average and reference value (Av-Ref) the goal is the 0 , because in this case the evaluated flatness is close to the real (or reference) value. In case of standard deviation of the evaluated flatness, the aim is to reach zero, because it ensures the good repeatability of the algorithm.

The minimum value of a repeated runs is very close to the reference value. The increasing size of the population and the number of mutated individuals improve the ratio, but the number of new individuals worsens it. In case of the average values of the repeated runs, the parameters have same effect, but the effect of the number of new individuals is clearer and the ration of average and reference value is larger. The standard deviation of the flatness values indicates the repeatability of the algorithm. The number of modified individuals has a large effect on it. The higher number of mutated items causes smaller standard deviation on a set of repeated runs. The increasing number of new individuals increases the standard deviation; the size of the population shows a reverse proportionality. With up to three modified individuals, the changes are small. 


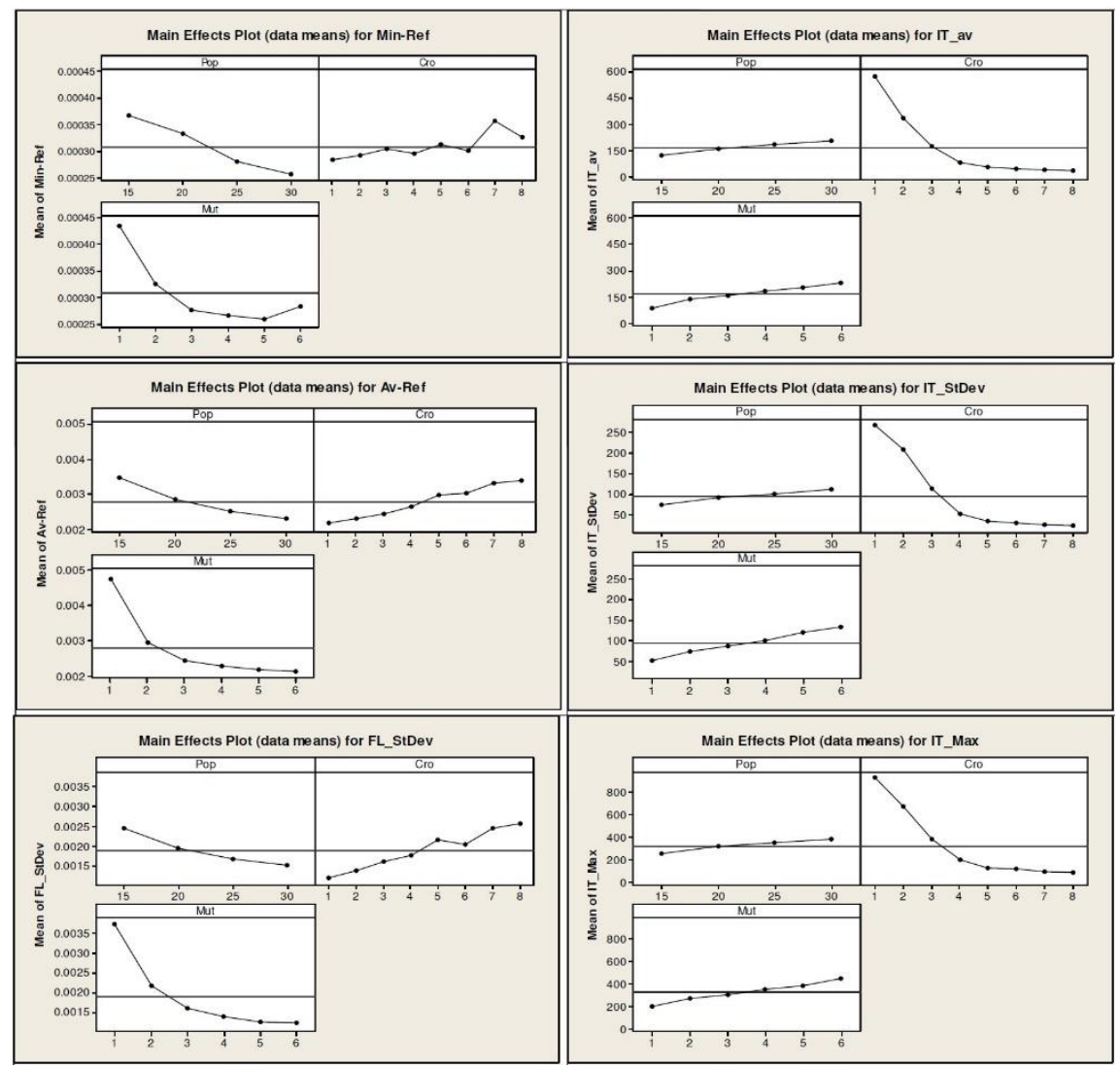

Figure 10

Main effects plots

The number of iterations until the stop condition indicates the speed of the algorithm. The average number of iteration, the maximum value and the standard deviation of the iteration are controlled by the number of new individuals, but up to 4 , the improvement is small. The size of the population and the number of mutated individuals increase the number of iteration cycles, but the effect is smaller.

Unfortunately, the two investigated sets of indicators show opposite nature. If a parameter improves the accuracy, the running time will be longer. If a parameter ensures faster execution, the accuracy of the results worsen.

During the optimization of GA parameters, the best set of Pop, Cro and Mut parameters should be determined, from the viewpoint of best performance. Several performance parameters can be selected. 
The "Minimum flatness - Reference flatness $=0$ " seems evident goal function, but the repeated runs got different flatness results, and if only one run is used, so there is no guarantee for the minimal flatness value.

Therefore, the "Average flatness - Reference flatness $=0$ " seems better, if the standard deviation of repeated runs is minimalized. The minimized standard deviation ensures the close range of results. Thus the accurate result can be calculated by a modification factor. The modification factor will consider the ratio between the average and the reference flatness. The number of iteration should be minimized, but the execution of the search is quite fast (less than one second), so it is not an important aspect.

Statistical analysis is performed to investigate the effects of GA parameters, i.e. Pop, Cro and Mut on the selected performance parameters. The main characteristics, which have important role in the performance, are the difference of Average flatness - Reference flatness $(A v-R e f)$ and the standard deviation of the repeated flatness determination (FL_StDev).

General linear model (GLM) is created for the statistical analysis for both output parameters. The purpose of this research is not only to describe the processes but also to optimize the setting of GA parameters on both performance parameters simultaneously. The statistical analysis is related to the above-mentioned factors and their levels:

- Pop: fixed factor with 4 levels

- Cro: fixed factor with 8 levels

- Mut: fixed factor with 8 levels

During the GLM analysis the two-way and the three-way interactions are taken into account. The optimization goals are to minimize the standard deviation of the repeated flatness determination and $A v-$ Ref value.

Table 3

ANOVA table for Av - Ref in case of the full data set $\left(\mathrm{R}^{2}=52.75 \%\right)$

\begin{tabular}{|l|r|r|r|r|r|}
\hline Source & \multicolumn{1}{|c|}{ DF } & \multicolumn{1}{c|}{ Adj SS } & Adj MS & F-Value & P-Value \\
\hline Pop & 3 & 0.000223 & 0.000074 & 38.56 & 0.000 \\
\hline Cro & 7 & 0.000214 & 0.000031 & 15.83 & 0.000 \\
\hline Mut & 5 & 0.000985 & 0.000197 & 102.08 & 0.000 \\
\hline Pop*Cro & 21 & 0.000057 & 0.000003 & 1.42 & 0.100 \\
\hline Pop*Mut & 15 & 0.000167 & 0.000011 & 5.78 & 0.000 \\
\hline Cro*Mut & 35 & 0.000360 & 0.000010 & 5.32 & 0.000 \\
\hline Pop*Cro*Mut & 105 & 0.000062 & 0.000001 & 0.31 & 1.000 \\
\hline Error & 960 & 0.001853 & 0.000002 & & \\
\hline Total & 1151 & 0.003922 & & & \\
\hline
\end{tabular}


It can be stated from the ANOVA table (Table 3) that the main factors (Pop, Cro, Mut) and the two-way interactions (Pop*Mut, Cro*Mut) have significant effect on the $A v-R e f$ value. This model has good fitting on the simulated values as the residuals show random distribution (Figure 11).
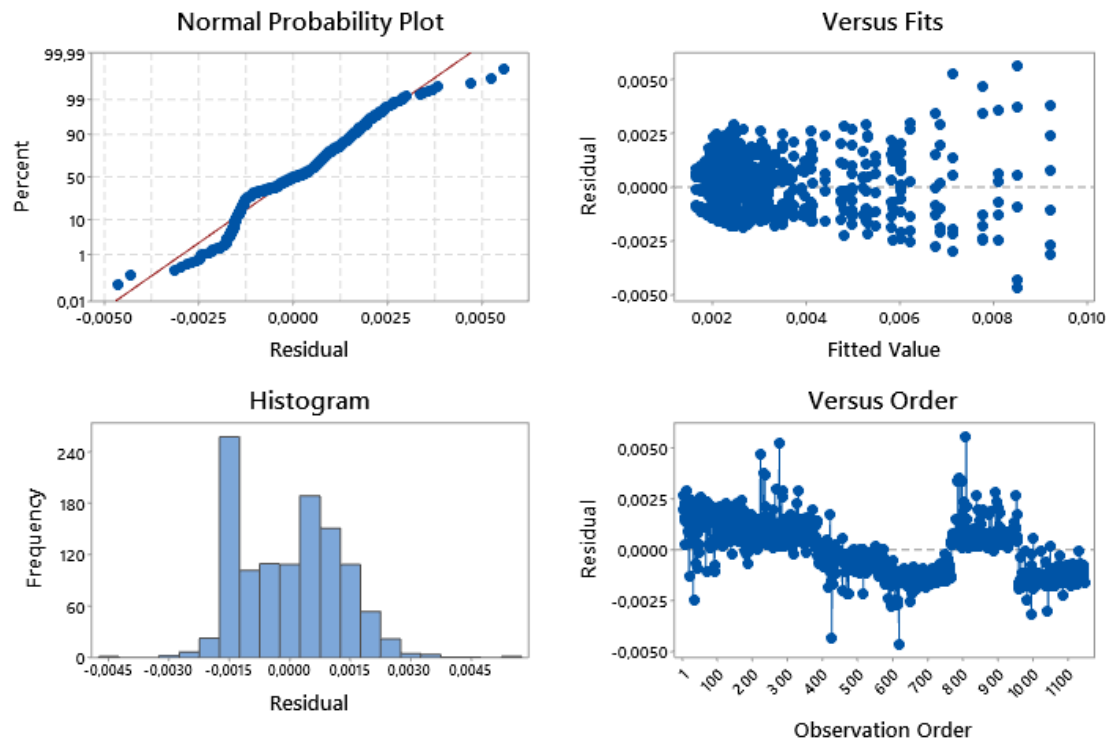

Figure 11

Residuals plots for the GLM of Av - Ref

The second GLM is related to the standard deviation of flatness determination. The results (Table 4) show that all main factors and two-way interactions have large effect on the output parameter. The residuals are randomly distributed (Figure 12).

Table 4

ANOVA table for FL_StDev in case of the full data set $\left(\mathrm{R}^{2}=58.04 \%\right)$

\begin{tabular}{|l|r|r|r|r|r|}
\hline Source & \multicolumn{1}{|c|}{ DF } & \multicolumn{1}{c|}{ Adj SS } & Adj MS & F-Value & P-Value \\
\hline Pop & 3 & 0.000142 & 0.000047 & 35.46 & 0.000 \\
\hline Cro & 7 & 0.000249 & 0.000036 & 26.67 & 0.000 \\
\hline Mut & 5 & 0.000892 & 0.000178 & 133.80 & 0.000 \\
\hline Pop*Cro & 21 & 0.000054 & 0.000003 & 1.91 & 0.008 \\
\hline Pop*Mut & 15 & 0.000100 & 0.000007 & 5.00 & 0.000 \\
\hline Cro*Mut & 35 & 0.000274 & 0.000008 & 5.86 & 0.000 \\
\hline Pop*Cro*Mut & 105 & 0.000061 & 0.000001 & 0.43 & 1.000 \\
\hline Error & 960 & 0.001280 & 0.000001 & & \\
\hline Total & 1151 & 0.003052 & & & \\
\hline
\end{tabular}



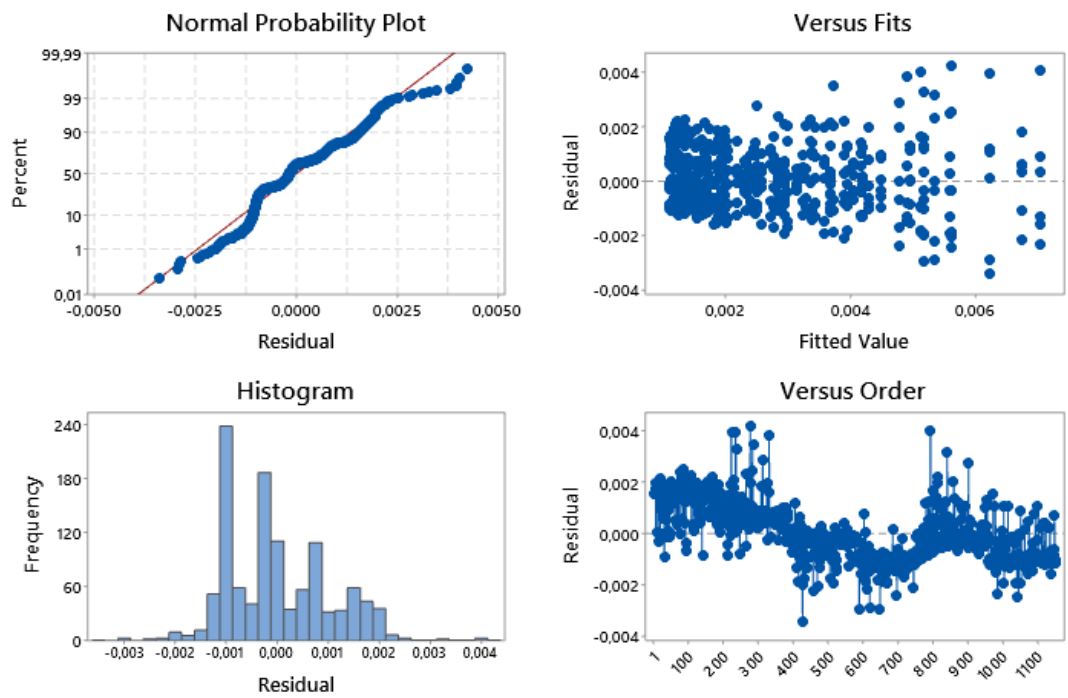

Figure 12

Residuals plots for the GLM of FL_StDev

During the optimization step, the two GLMs are optimised simultaneously. Within this models only the GA parameters and their interactions are taken into account. The goal is to minimize $A v-\operatorname{Ref}$ and the FL_StDev values. In case of all test parts the optimum parameters of the genetic algorithm are $\mathrm{Pop}=25, \mathrm{Cro}=7$ and Mut=5 (Figure 13). The reached $A v-R e f$ value is $1.6 \mu \mathrm{m}$, the standard deviation for flatness determination is $1.2 \mu \mathrm{m}$.

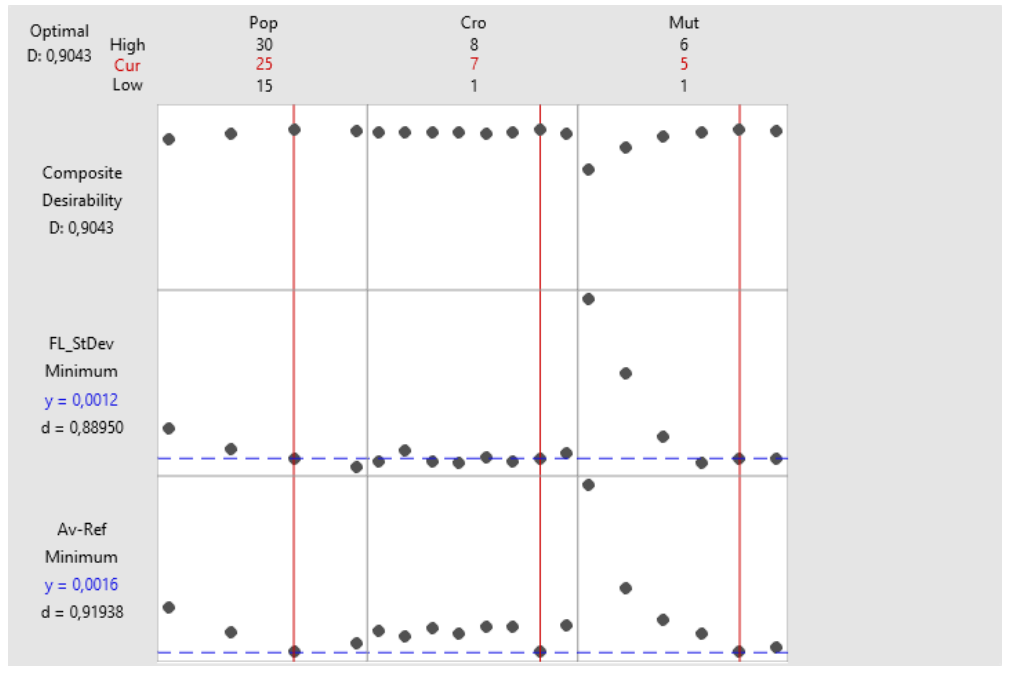

Figure 13

Multiple response ( $A v$ - Ref and FL_StDev) optimizer for the data 
If the data of the test parts are viewed as individual sets and the surface IDs are taken into account with its 6 levels as a main factor, the ANOVA results for $A v-$ Ref values (Table 5) and for FL_StDev values (Table 6) are better.

Table 5

ANOVA table for $A v-R e f$ in case of the full data set with surface ID main factor $\left(\mathrm{R}^{2}=88.83 \%\right)$

\begin{tabular}{|l|r|c|c|r|r|}
\hline Source & \multicolumn{1}{|c|}{ DF } & \multicolumn{1}{c|}{ Adj SS } & Adj MS & F-Value & P-Value \\
\hline Pop & 3 & 0.000223 & 0.000074 & 162.31 & 0.000 \\
\hline Cro & 7 & 0.000214 & 0.000031 & 66.64 & 0.000 \\
\hline Mut & 5 & 0.000985 & 0.000197 & 429.70 & 0.000 \\
\hline Surface ID & 5 & 0.001415 & 0.000283 & 617.22 & 0.000 \\
\hline Pop*Cro & 21 & 0.000057 & 0.000003 & 5.97 & 0.000 \\
\hline Pop*Mut & 15 & 0.000167 & 0.000011 & 24.33 & 0.000 \\
\hline Cro*Mut & 35 & 0.000360 & 0.000010 & 22.40 & 0.000 \\
\hline Pop*Cro*Mut & 105 & 0.000062 & 0.000001 & 1.29 & 0.033 \\
\hline Error & 955 & 0.000438 & 0.000000 & & \\
\hline Total & 1151 & 0.003922 & & & \\
\hline
\end{tabular}

Table 6

ANOVA table for FL_StDev in case of the full data set with surface ID main factor $\left(\mathrm{R}^{2}=86.71 \%\right)$

\begin{tabular}{|l|r|r|r|r|r|}
\hline Source & \multicolumn{1}{|c|}{ DF } & \multicolumn{1}{c|}{ Adj SS } & Adj MS & F-Value & P-Value \\
\hline Pop & 3 & 0.000142 & 0.000047 & 111.34 & 0.000 \\
\hline Cro & 7 & 0.000249 & 0.000036 & 83.75 & 0.000 \\
\hline Mut & 5 & 0.000892 & 0.000178 & 420.15 & 0.000 \\
\hline Surface ID & 5 & 0.000875 & 0.000175 & 411.93 & 0.000 \\
\hline Pop*Cro & 21 & 0.000054 & 0.000003 & 6.01 & 0.000 \\
\hline Pop*Mut & 15 & 0.000100 & 0.000007 & 15.71 & 0.000 \\
\hline Cro*Mut & 35 & 0.000274 & 0.000008 & 18.42 & 0.000 \\
\hline Pop*Cro*Mut & 105 & 0.000061 & 0.000001 & 1.36 & 0.013 \\
\hline Error & 955 & 0.000406 & 0.000000 & & \\
\hline Total & 1151 & 0.003052 & & & \\
\hline
\end{tabular}

The multiple response optimization is performed for the six surfaces separately.

Table 7 shows the results.

Table 7

Multiple response ( $A v$ - Ref and $\left.F L \_S t D e v\right)$ optimizer for the data grouping by surfaces

\begin{tabular}{|l|c|c|c|c|c|c|}
\hline $\begin{array}{c}\text { Parameter/Surface } \\
\text { ID }\end{array}$ & Sf\#1 & Sf\#2 & Sf\#3 & Sf\#4 & Sf\#5 & Sf\#6 \\
\hline Pop & 30 & 25 & 25 & 30 & 25 & 30 \\
\hline Cro & 7 & 7 & 7 & 1 & 7 & 1 \\
\hline Mut & 6 & 5 & 5 & 2 & 5 & 2 \\
\hline
\end{tabular}




\begin{tabular}{|l|l|l|l|l|l|l|}
\hline Av-Ref [mm] & 0.0031 & 0.0027 & 0.0012 & 0.0005 & 0.0024 & 0.0005 \\
\hline FL_StDev [mm] & 0.0025 & 0.0021 & 0.0009 & 0.0001 & 0.0013 & 0.0002 \\
\hline
\end{tabular}

When the data examined separately, the optimal GA parameters are different. In case of Sf\#2, Sf\#3 and Sf\#5, the parameters are the same, and in case of Sf\#1 they are close to the overall parameters (25/7/5). Nevertheless, in case of Sf\#4 and Sf\#6 the number of crossover (Cro) and the number of mutation (Mut) is very different. These separated parameters can ensure better performance of the genetic algorithm, but in order for a general application, the nature of the investigated surface cannot be considered.

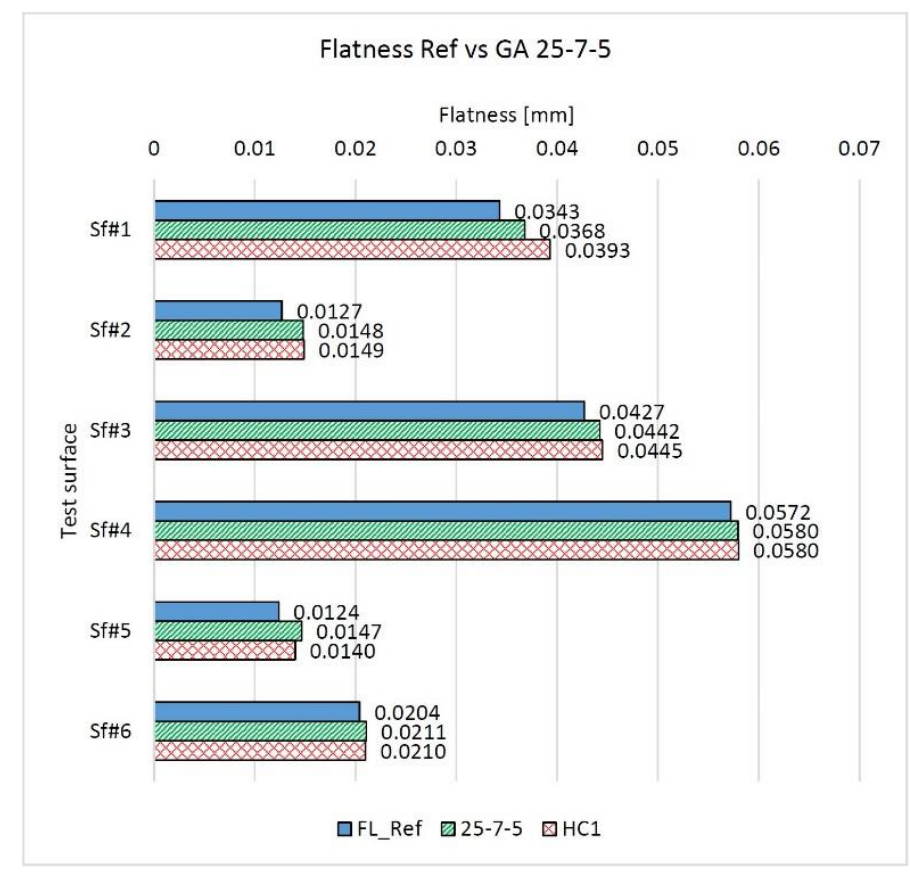

Figure 14

Comparison of flatness results

Figure 14 shows the results of the optimised genetic algorithm (25-7-5) comparing with the reference values (FL_Ref) and the results of random hill climbing algorithm (HC1). Generally, the optimized genetic algorithm achieves better results, but sometimes the differences are very small, less than $1 \mu \mathrm{m}$.

\section{Conclusions}

The description of a machine part requires not only the shape, the size or the material, but also, the tolerances. The geometric tolerances ensure a more sophisticated definition of shape and position deviations, but the use of coordinate metrology is necessary. The flatness describes the deviation of the machined 
surface from a theoretical plane. However, the standards define the interpretation of flatness, but the mathematical evaluation process can be different. Different types of methods can be used, such as, the least square method and the minimum zone method of enveloping methods. In addition, the mathematical implementations can be very different.

In this work, the minimum zone method is presented, and the application of genetic algorithm (GA) is investigated. The GA has three basic parameters, which characterize the work of the algorithm. These parameters are (1) the size of the population (Pop); (2) the number of new individuals, which are created by crossover operator (Cro); and (3) the number of individuals, which are modified by mutation operator (Mut). These parameters were optimized based on measurement results of test surfaces. The size of the tests surfaces was $175 \times 155$ $\mathrm{mm}$, and they were machined by milling or turning methods. The test point clouds contain 1020 coordinate points. The flatness values of genetic algorithm were compared with hill-climbing algorithm.

The most important statements of this research are:

- In case of the presented implementation, the genetic algorithm is able to solve the flatness evaluation problem considering the minimum zone method. Comparing with the previous results, the time of the evaluation is similar (less, than $1 \mathrm{~s}$ ).

- The parameters of genetic algorithm can be optimized by statistical method, and in the investigated case the optimal parameters are $\mathrm{Pop}=25$, $\mathrm{Cro}=7$, Mut=5.

- The properties of the investigated surfaces have effect on the GA parameters, although the general use of the GA method does not allow these to be taken into account.

- The calculated flatness values of the optimized genetic algorithm generally better than the results of the hill climbing algorithm in case of the investigated surfaces. But the differences are very small, less than $1 \mu \mathrm{m}$.

In future research, the effects of surface parameters could be investigated, such as, the size, the machining technology and the surface roughness. Another task could be the application of other types of swarm algorithms, like bee colony, grey wolf optimizer, cuckoo search etc.

\section{References}

[1] ISO 1101-2017 Geometrical product specifications (GPS) - Geometrical tolerancing - Tolerances of form, orientation, location and run-out.

[2] ASME Y14.5-2018 (2018) Dimensioning and tolerancing. ASME

[3] B Štrbac; B Ačko; S Havrlišan; I Matin; B Savković; M Hadžistević (2020) Investigation of the effect of temperature and other significant factors on 
systematic error and measurement uncertainty in CMM measurements by applying design of experiments. Measurement 158(1):107692, DOI: 10.1016/j.measurement.2020.107692

[4] A. M. Abdulshahed; I. Badi; A. Alturas (2019) Efficient evaluation of flatness error from coordinate measurement data using Cuckoo search optimisation algorithm. Journal of Academic Research 6:37-51

[5] Y. Yang; M. Li; J-J. Gu (2019) Application of adaptive hybrid teachinglearning-based optimization algorithm in flatness error evaluation. Journal of Computers 30(4):63-77, DOI: 10.3966/199115992019083004006

[6] T. Kanada; S. Suzuki (1993) Evaluation of minimum zone flatness by means of nonlinear optimization techniques and its verification. Precision Engineering 15(2):93-99, DOI: 10.1016/0141-6359(93)90343-9

[7] G. L. Samuel; M. S. Shunmugam (1999) Evaluation of straightness and flatness error using computational geometric techniques. Computer-aided Design 31(11):829-843, DOI: 10.1016/S0010-4485(99)00071-8

[8] Hermann Gy. (2007) Robust convex hull-based algorithm for straightness and flatness determination in coordinate measuring. Acta Polytechnica Hungarica 4(4):111-120

[9] H-Y. Tseng (2006) A genetic algorithm for assessing flatness in automated manufacturing systems. J Intelligent Manufacturing 17:301-306, DOI: 10.1007/s10845-005-0004-y

[10] D-X. Wang; X-L. Wen; F-L. Wang (2012) A differential evolutionary algorithm for flatness error evaluation. AASRI Procedia 1:238-243, DOI: 10.1016/j.aasri.2012.06.037

[11] X-L. Wen et al. (2012) Flatness error evaluation and verification based on new generation geometrical product specification (GPS). Precision Engineering 36(1):70-76, DOI: 10.1016/j.precisioneng.2011.07.006

[12] M. I. Khan; S. Ma (2014) Efficient genetic algorithms for measurement of flatness error and development of flatness plane based on minimum zone method. Advanced Materials Research 941-944:2232-2238, DOI: 10.4028/www.scientific.net/AMR.941-944.2232

[13] B. Štrbac; B. Mikó; D. Rodić; J. Nagy; M. Hadžistević (2020) Analysis of characteristics of non-commercial software systems for assessing flatness error by means of minimum zone method. Tehnički Vjesnik 27(2):535-541, DOI: $10.17559 / \mathrm{TV}-20190603084835$

[14] X. Wen; Q. Xia; Y. Zhao (2006) An effective genetic algorithm for circularity error unified evaluation. International Journal of Machine Tools \& Manufacture 46(14):1770-1777, DOI: 10.1016/j.ijmachtools.2005.11. 015 
[15] A. Rossi; M. Antonetti; M. Barloscio; M. Lanzetta (2011) Fast genetic algorithm for roundness evaluation by the minimum zone tolerance (MZT) method. Measurement 44(7):1243-1252, DOI: 10.1016/j.measurement. 2011.03.031 